Gynäk. Rdsch. 1974;14(Suppl. 1):1-2

\title{
Contents, Vol. 14, Supplement 1, 1974
}

\section{/. Hauptthema}

Bestimmung des intrauterinen Reifegrades des Kindes

ler sujet principal

Determination intra-utérine du degré de maturité de Гenfant

Hinselmann, M. (Basel): Ultraschallmethoden

Kubli, F.; Lorenz, U. und Rüttgers, H. (Heidelberg): Fruchtwasseruntersuchungen zur intrauterinen Reifegradbestimmung 10

Watteville, H. de et Rosenblatt, R. (Geneve): L'évaluation de la maturité fæate par $\Gamma$ étude du frottis vaginal et $\Gamma$ examen cytologique du liquide amniotique . 21 Stamm, O.;

Latscha, U.; Janecek, P. und Campana, A. (St. Gallen): Kontinuier-

liche subkutane $\mathrm{pH}-$ Messung am kindlichen Kopf sub partu und post partum 28

Mitteilungen zum 1. Hauptthema Communications se rapportant au ler sujet principal

De Grandi, P.; Lüthi, A.; Eppenberger, U.; Ramzin, M. et Hinselmann, M.

(Bale): Concentration d'amylase dans le liquide amniotique et degré de maturité

fæata 36

Béguin, F. et Arendt, J. (Geneve): Concentrations de Tacide 5-hydroxy-indole-

acétique dans le liquide amniotique et maturité fætale

Baur, B.; Moser, R. und Sidiropoulos, D. (Bern): Retrospektive Studie über den Aussagewert der ultrasonographischen Kephalometrie zur indirekten Bestim mung des RDS-Risikos

Cruz, J. M. et Bossart, H. (Lausanne): Croissance du diametre bipariétal fætal en function de différents paramètres maternels 44

Modly, T.; Zürcher, W. O. und Hinselmann, M. (Basel): Vergleichende Terminbestimmung mittels Ultraschall und Röntgenbild (Schwangerschaftsübersichtsaufnahme) $\quad 45$

Kunz, J.; Keller, P. J. und Schmid, J. (Zurich): Ultraschallbefunde und Östriolwerte bei intrauteriner Mangelentwicklung 46

2

Index

Brunner, J.; Schwyter, H.; Kunz, J.; Keller, P. J.; Schmid, J. und Mieth, D.

(Zurich): Phospholipide im Fruchtwasser 49

Schmid, J.; Schwyter, H.; Kunz, J. und Keller, P. J. (Zurich): Veränderungen der Phospholipide im Fruchtwasser unter Kortisongabe

Engelhorn, A. et Berli, Ch. (Geneve): Le dosage des phospholipides dans le liquide amniotique et la maturité fcetale 53

Dubler, O. und Schmid, R. (Münsterlingen): Aussagekraft des Clements-Tests bei der Bestimmung der fetalen Lungenreife 55

Dreher, E. (Basel): Austausche von Sphingomyelin und Lezithin zwischen Mutter und Fetus 58 
Haag, B.; Decker, K.; Hinselmann, M. und Hirsch, H. A. (Basel): Bakteriologische Befunde und Infektionsgefährdung bei diagnostischer Fruchtwascerpunktion 59 Hammacher, K.; Brun del Re, R.; Gaudenz, R.; De Grandi, P. und Richter, R. (Basel): Kardiotokographischer Nachweis einer fetalen Gefährdung mit einem CTG-Score 61

Richter, R.; Brun del Re, R.; De Grandi, P.; Hammacher, K. und Hindemann, P. (Basel): Klinische Bedeutung der Reifegradbestimmung bei kardiotckographischem Hinweis auf eine fetale Gefährdung 64

O $\tau \tau$, A. (Winterthur): Fetale Reifebestimmung bei kongenitalem Adrenogenitalsyndrom der Mutter 67

Baertschi, U. und Schmid, J. (Zurich): Reifegradbestimmung und Wahl des Geburtstermins bei Risikoschwangerschaften $\quad 70$

2. Hauptthema Sterilität, hauptsächlich Zervixfaktor 2e sujet principal Stérilité, en particulier facteur cervical

Wenner, R. und Obolensky, W. (Liestal): Zervixfaktor in der Sterilität

Schirren, C. (Hamburg-Eppendorf): Andrologische Aspekte der Sterilität

Maroni, E. (Zurich): Unverträglichkeit von Sperma und Frau

Hauser, G. A.; Samartzis, S. und Ackermann, U. (Luzern): Zustand der Kinder nach erfolgreich behandelter Sterilität 97

Mitteilungen zum 2. Hauptthema Communications se rapportant au 2e sujet principal Schmid-Tannwald, I.: Samartzis, S. und Hauser, G. A. (Luzern): Virginität in der Ehe als Sterilitätsursache 107

Berger, M. und Meszaros, Th. (Bern): Tubenplastik mit gestieltem Myometriumlappen 109

Balmer, J. A. (Bern): Beeinflussung der Spermienmotilität 111 Schweizerische Gesellschaft für Gynäkologie

Bericht über die Jahresversammlung, 20.-22. Juni 1974 in Luzern

Société Suisse de Gynécologie

Compte rendu de ГAssemblée annuelle, 20-22 juin 1974 à Lucerne Redaktor - Rédacteur: H.

Erb, Liestal

1974;014:1 